\title{
Helicobacter cinaedi bacteraemia secondary to enterocolitis in an immunocompetent patient
}

\author{
Sofie Larsen Rasmussen ${ }^{1}$, Iben Ørsted ${ }^{2}$, Irene Harder Tarpgaard ${ }^{1}$ and Hans Linde Nielsen ${ }^{1,3^{*}}$ [D
}

\begin{abstract}
Background: Helicobacter cinaedi are motile, gram-negative spiral rods with a natural reservoir in the intestinal tract of hamsters and rhesus monkeys. In humans, H. cinaedi has been reported in different human infections like fever, abdominal pain, gastroenteritis, proctitis, diarrhoea, erysipelas, cellulitis, arthritis, and neonatal meningitis typically diagnosed by positive blood cultures. Even though $\mathrm{H}$. cinaedi has been detected from human blood and stool the entry of $\mathrm{H}$. cinaedi into the blood stream was undocumented until quite recently. The use of pulse-field gel electrophoresis (PFGE) demonstrated that stool- and blood-derived H. cinaedi strains were consistent.
\end{abstract}

Case presentation: Here, we describe a rare Danish case of $\mathrm{H}$. cinaedi bacteraemia in an immunocompetent 44-yearold male with diarrhoea. We isolated $\mathrm{H}$. cinaedi from a blood culture taken at admission, and from a FecalSwab taken at day six despite ongoing antibiotic therapy. Next, we made a genetic comparison of both isolates by use of Multilocus sequence typing (MLST)- and Single nucleotide polymorphism (SNP)-analysis. The two isolates were identical with zero SNPs and by use of MLST the isolate was identified as a novel ST20, confirming previous data of the intestinal tract as a route of $\mathrm{H}$. cinaedi bacteraemia. The results of our AST showed a resistance pattern with higher MICs for ciprofloxacin and clarithromycin than for ampicillin, amoxicillin, gentamicin, and imipenem. The patient was cured with targeted therapy with pivampicillin; however, the primary source of transmission was unknown.

Conclusions: In conclusion, this case of $\mathrm{H}$. cinaedi bacteraemia secondary to enterocolitis in an immunocompetent patient provide clear evidence that one route of infection occurs through translocation from the intestinal tract to the bloodstream. Helicobacter cinaedi from blood and faeces were identical with a novel ST20, resistant to ciprofloxacin and clarithromycin however, the patient was cured with oral pivampicillin.

Keywords: Helicobacter cinaedi, Bacteraemia, Gastroenteritis, Enterocolitis, MLST, SNP-analysis

\section{Background}

Helicobacter cinaedi was originally described as Campylobacter cinaedi by Totten et al. in 1985 however, six year later it was reclassified into genus Helicobacter by Vandamme et al. [1, 2]. These organisms are motile, gram-negative spiral rods which have been isolated from different domestic animals including dogs, cats and foxes [3]. However, the only natural reservoir of $H$. cinaedi is

\footnotetext{
*Correspondence: halin@rn.dk

${ }^{1}$ Department of Clinical Microbiology, Aalborg University Hospital, Hobrovej 18, 9000 Aalborg, Denmark

Full list of author information is available at the end of the article
}

the intestinal tract of hamsters and rhesus monkeys [3, 4].

In humans, $H$. cinaedi was first isolated from the rectal cultures of HIV-positive, homosexual men with proctitis, proctocolitis, and enteritis [5]. It was initially considered an opportunistic pathogen that caused disease in immunocompromised patients however in recent years infections in immunocompetent patients have also been reported [6]. Most $H$. cinaedi infections with symptoms such as fever, abdominal pain, gastroenteritis, proctitis, diarrhoea, erysipelas, cellulitis, arthritis and neonatal meningitis have been identified by positive blood cultures [5-8]. 
Even though $H$. cinaedi was detected in both blood and stool cultures the entry of $H$. cinaedi into the blood stream was undocumented for decades. However, in 2018 Araoka et al. demonstrated by use of pulse-field gel electrophoresis (PFGE) that stool- and blood-derived $H$. cinaedi strains were consistent in nine Japanese patients giving first evidence of translocation from the intestinal tract as one of the routes that leads to $H$. cinaedi bacteraemia [9].

Here, we describe a rare Danish case of $H$. cinaedi bacteraemia in an immunocompetent patient with diarrhoea. We isolated $H$. cinaedi from blood and faeces and made a genetic comparison of the isolates by use of Multi-locus sequence typing (MLST)- and Single nucleotide polymorphism (SNP)-analysis and found that they were identical, confirming previous data of the intestinal tract as a route of $H$. cinaedi bacteraemia [9].

\section{Case presentation}

A 44-year-old male with a family history of polycystic kidney disease though otherwise healthy with no medical record was admitted to Aalborg University Hospital on suspicion of chronic kidney disease. The patient presented with nausea and diarrhoea with five lose watery stools a day for two weeks, and a sudden onset of fever, chills, flank and lower back pain, and dark urine. He did not report any bloody diarrhoea, nor dysuria, polyuria, urgency or haematuria. His temperature was $39.8{ }^{\circ} \mathrm{C}$ however, the physical examination and his vital parameters were normal. Laboratory findings showed leucocytosis with a WBC-count of $17.5 \times 10^{9} / \mathrm{L}$, an elevated CRP of $90 \mathrm{mg} / \mathrm{L}$, and a mild hyponatremia (p-sodium: $136 \mathrm{mmol} / \mathrm{L}$ ) and hypokalaemia (p-potassium: $3.4 \mathrm{mmol} / \mathrm{L}$ ) however, there was a normal $\mathrm{p}$-creatinine of $80 \mu \mathrm{mol} / \mathrm{L}$. Urine and blood culture was obtained, and on suspicion of urinary tract infection standard empiric antibiotic therapy was initiated with intravenous ampicillin $2 \mathrm{~g}$ every $6 \mathrm{~h}$, and gentamicin $5 \mathrm{mg} / \mathrm{kg}$ once daily. After two days the patient was afebrile nevertheless, CRP was further elevated to 301. Therefore, the antibiotic therapy was changed to intravenous mecillinam (amdinocillin) $1 \mathrm{~g}$ every $8 \mathrm{~h}$. The chest $\mathrm{x}$-ray was normal however, ultrasound showed multiple cysts in the liver and in both kidneys however, no sign of hydronephrosis.

Urine culture was negative. On day six there was a positive blood culture with growth of Gram-negative spiral-shaped rods and on preliminary suspicion of Campylobacter bacteraemia oral roxithromycin $150 \mathrm{mg}$ twice daily was initiated. In addition, a stool culture was sent to the laboratory. Seven days after hospitalization he was afebrile, CRP had dropped to $133 \mathrm{mg} / \mathrm{L}$. The diarrhoea symptoms were declining, and still he had no urinary symptoms, so he was discharged with oral pivmecillinam $400 \mathrm{mg}$ every $8 \mathrm{~h}$ and roxithromycin. At day 10 the patient was seen in an outpatient setting and he reported ongoing diarrhoea and with a simultaneous identification of $H$. cinaedi in blood and stools antibiotic therapy was changed to oral pivampicillin $500 \mathrm{mg}$ every $8 \mathrm{~h}$ for seven days. At day 17, he no longer had diarrhoea and CRP was normalised at $5.7 \mathrm{mg} / \mathrm{L}$. The patient owned a dog, and had no close contact to other animals and was immunocompetent.

\section{Investigations}

A standard blood culture was obtained upon submission (Two BD BACTEC ${ }^{\mathrm{TM}}$ Plus Aerobic medium and one BD BACTEC $^{\mathrm{TM}}$ Lytic Anaerobic medium glass culture vials) incubated in the BACTEC FX Top instrument (Becton Dickinson AB, Stockholm, Sweden). After six days of incubation there was growth in the two aerobic bottles (Time to detection (TTD): 141.3 and $143.6 \mathrm{~h}$, respectively) of motile, Gram-negative, spiral-shaped rods. Subcultivation was performed on $5 \%$ yeast-enriched horse blood agar plates (SSI Diagnostica, Hillerød, Denmark) at $37{ }^{\circ} \mathrm{C}$ in a hydrogen-enriched microaerobic atmosphere $\left(6 \% \mathrm{O}_{2}, 6 \% \mathrm{CO}_{2}, 6 \% \mathrm{H}_{2}\right.$, and $\left.82 \% \mathrm{~N}_{2}\right)$ and examined after $48 \mathrm{~h}$. The isolate was urease negative though oxidase and catalase positive, and final identification of $H$. cinaed $i$ was performed by use of the matrix-assisted laser desorption ionization-time of flight (MALDI Biotyper 3.1, Bruker Daltonics Microflex LT, MBT 6903 MSP Library) with a score of 2.060 .

No clinical breakpoints exist for $H$. cinaedi, however, antibiotic susceptibility testing (AST) was performed by use of McFarland standard 1.0 on Mueller-Hinton agar for fastidious organisms ( $\mathrm{MH}$ supplemented with 5\% defibrinated horse blood and $20 \mathrm{mg} / \mathrm{L} \beta$-NAD) and Etest (BioMérieux, Marcy l'Etoile, France) in a microaerobic atmosphere. The isolate showed low minimum inhibitory concentrations (MICs) to ampicillin (MIC: $2 \mathrm{mg} / \mathrm{L}$ ), amoxicillin (MIC: $4 \mathrm{mg} / \mathrm{L}$ ), imipenem (MIC: $0.032 \mathrm{mg} / \mathrm{L}$ ) and gentamicin (MIC: $0.25 \mathrm{mg} / \mathrm{L}$ ) and resistant to ciprofloxacin (MIC: $>32 \mathrm{mg} / \mathrm{L}$ ) and clarithromycin (MIC: $>256 \mathrm{mg} / \mathrm{L}$ ). For mecillinam (no etest available) we included disc diffusion (disc content, $10 \mu \mathrm{g}$ ), showing an inhibition zone of $18 \mathrm{~mm}$, however, we were unable to interpret this result.

After the positive blood culture on day 6 a FecalSwab (COPAN ITALIA, Brescia, Italy) was sent to the laboratory. The sample was negative for Aeromonas, Campylobacter, Salmonella, Shigella, Vibrio, Yersinia enterocolitica by routine culture methods and Clostridioides difficile toxins by use of the Xpert $C$. difficile BT (Cepheid). However, $0.5 \mathrm{ml}$ liquid media from the FecalSwab were incubated by use of a polycarbonate filter method on a $5 \%$ yeast-enriched blood agar plate (SSI 
Diagnostica, Hillerød, Denmark) as described elsewhere [10]. After $48 \mathrm{~h}$ at $37{ }^{\circ} \mathrm{C}$ in a hydrogen-enriched microaerobic atmosphere, there was growth of $H$. cinaedi, identified with a MALDI log-score of 2.080.

Next, we performed genome sequencing of both isolates to investigate whether the $H$. cinaedi in faeces and blood were identical. We used the Illumina MiSeq instrument producing $2 \times 300$-bp paired-end reads by using Nextera XT library preparation kit (Illumina Denmark ApS, Copenhagen, Denmark). Reads were assembled using CLC Genomics Workbench (version 11) (QIAGEN Bioinformatics, Aarhus, Denmark) into contigs $(\mathrm{n}=74+\mathrm{n}=72 \geq 1 \mathrm{~kb}$, respectively), N50 $(89,940+96,798$, respectively), total sequence length $2,406,086+2,403,138 \mathrm{bp}$, respectively, and with a G+C content of $38.4 \%$. To subtype our strains and identify sequence type (ST) we used the $H$. cinaedi Multilocus Sequence Typing (MLST) database [11]. Both isolates were classified as a novel ST20 (allelic profile, 23S_rRNA: 5, ppa: 7, aspA: 5, aroE: 7, atpA: 3, tkt: 5, cdtB: 3) (The blood isolate, AAUH 190,263, was uploaded to: https:// pubmlst.org/). Next, we used the CSI Phylogeny v1.4 pipeline (with default-settings) for SNP-calling between isolates [12], which showed 0 SNPs, giving clear evidence of identical strains and a translocation of $H$. cinaedi from the intestinal tract into the bloodstream.

\section{Discussion and conclusions}

This is the first Danish case of $H$. cinaedi bacteraemia with documented evidence of translocation from the intestinal tract in an immunocompetent patient. We isolated $H$. cinaedi from blood in two aerobic BACTEC bottles after six days of incubation, and faeces by use of the polycarbonate filtration technique. By use of MLST and SNP-analyses we identified the two isolates as a single (zero SNPs) ST20. Araoka et al. evaluated the TTD for $H$. cinaedi in the BACTEC system and found median time of 5 days (range, 2 to 12 days), and all $H$. cinaedi strains (126 positive sets) were detected in aerobic bottles only [7]. The blood cultures in our laboratory are routinely incubated for 7 days, which theoretically should be sufficient to find $87 \%$ of 'true' $H$. cinaedi-positive blood cultures [7]. In comparison, Kawamura et al. also reported positive results with the VersaTREK systems providing excellent growth ability for $H$. cinaedi [8] however, to our knowledge the VersaTREK system is not available in Denmark.

Helicobacter cinaedi infection seems very uncommon in Denmark, with only two previous cases: One case with blood stream infection secondary to cellulitis, and one case with septic arthritis, respectively [13, 14]. Nielsen et al. investigated 5.963 diarrheic stool samples from 4.094 patients and identified two isolates of $H$. cinaedi by use of the polycarbonate filter method [10]. Thus, $H$. cinaedi is uncommon in Danish stool samples. In contrast, $H$. cinaedi has been isolated from diarrheic stool samples and blood cultures of paediatric patients in South Africa [15], and it has been reported that $H$. cinaedi constitutes approximately $2.2 \%$ of positive blood cultures in Japan [7]. Recurrent bacteraemia has also been described with cellulitis as the most frequent clinical symptom [16-19]. The cause of the substantial difference in occurrence of $H$. cinaedi infection between Denmark and Japan is unknown.

Through genome sequencing we confirmed that the isolates of $H$. cinaedi from stool and blood were identical by MLST-profile and zero SNPs. Previously, MLST were used to identify $H$. cinaedi STs causing nosocomial outbreaks in Japanese hospitals, whereas some isolates were non-typeable by Pulsefield gel electrophoresis PFGE [11]. In the MLST-database the majority of $H$. cinaedi isolates $(\mathrm{n}=70$ per 2021-02-10) are from Japan (89\%), USA or Australia, so the designation of a 'novel' ST is not surprising. Our isolate is also the single European $H$. cinaedi isolate in the database.

Until recently, the entrance of $H$. cinaedi into the blood stream were more or less undocumented and Kawamura et al. stated the importance of pursuing the route of infection [8]. In 2018, Araoka et al. described PFGE typing to confirm clonality of the faeces and blood isolates from nine immunocompromised patients with fever and cellulitis, delivering the first evidence of bacterial translocation from the intestinal tract as a route of $H$. cinaedi bacteraemia [9]. Our patient did not show any signs of cellulitis in addition to diarrhoea, nor was he immunocompromised, whereas the nine patients described by Araoka et al. were treated with either steroids or anticancer chemotherapy [9]. Interestingly, the same study group identified anticancer chemotherapy and systemic steroids as independent risk factors for recurrent $H$. cinaedi bacteraemia [16], a condition that apparently seems rare outside of Japan. Previous case reports have also described hepatic and kidney cyst infection with bacteraemia caused $H$. cinaedi [20-22], however the radiological imaging showed no sign of infection in the cysts in the presented case.

Helicobacter cinaedi is not known to normally colonize the intestines of humans, and it has been proposed that infection might develop through zoonotic transmission [8], or through nosocomial spread among immunocompromised patients during hospitalization [23, 24]. Our patient owned a dog, and had no close contact with other animals, so the primary source of transmission remains unknown. Kiehlbauch et al. showed that $H$. cinaedi isolates from human, dog, and hamster formed distinct ribotype pattern [25], and two decades later Kawamura et al. found that isolates from dogs which had been identified as $H$. cinaedi 
were different from human $H$. cinaedi isolates by wholegenome in silico DNA similarities and therefore re-classified as the new Helicobacter canicola [26].

There is no clinical breakpoints nor recommended guideline for the choice or duration of antibiotic therapy for $H$. cinaedi infection. Recently, Nukui et al. suggested a twoweek antibiotic therapy to prevent recurrence of $H$ cinaed $i$ bacteremia [27]. The results of our AST were in coherence with previously reported resistance patterns of $H$. cinaedi with higher MICs for ciprofloxacin and clarithromycin than for ampicillin, amoxicillin, gentamicin, and imipenem $[8,27]$. After admission, the patient responded on empiric therapy with ampicillin and gentamicin and became afebrile. However, the empiric antibiotic regime was later changed to intravenous mecillinam, and despite this therapy $H$. cinaedi could be isolated from a FecalSwab taken six days after admission. Pivmecillinam and roxithromycin had also no clinical effect as diarrheal symptoms resided. However, a change to pivampicillin stopped the diarrheal symptoms and CRP normalized, and to our knowledge the patient has not been hospitalized since.

In conclusion, this case of $H$. cinaedi bacteraemia secondary to enterocolitis in an immunocompetent patient provide clear evidence that one route of infection occurs through translocation from the intestinal tract to the bloodstream. Helicobacter cinaedi from blood and faeces were identical with a 'novel' ST20, resistant to ciprofloxacin and clarithromycin however, the patient was cured with oral pivampicillin.

\section{Abbreviations}

AST: Antibiotic susceptibility testing; MIC: Minimum inhibitory concentration; MLST: Multi-locus sequence typing; PFGE: Pulse-field gel electrophoresis; ST:

Sequence type; SNP: Single nucleotide polymorphism; TTD: Time to detection.

\section{Acknowledgements}

The Authors would like to thank Senior Researcher Rolf Sommer Kaas from Center for Genomic Epidemiology, DTU, Kgs. Lyngby, Denmark for his help with interpretation of the SNP-analysis.

\section{Authors' contributions \\ HLN made the first design of the manuscript, isolation of the bacteria, identification, and WGS-analysis. IHT made the DNA extraction and library preparation for sequencing. SLR drafted the manuscript, and IØ treated the patient and obtained informed consent from the patient. All Authors revised the manuscript critically and approved the final version. All authors have read and approved the final manuscript.}

\section{Funding}

The manuscript was made without external funding.

\section{Availability of data and materials}

The MLST allelic profile and sequence for isolate AAUH 190,263 is available at https://pubmlst.org/ Additional WGS-data is available on specific request to the corresponding Author.

\section{Declarations}

Ethics approval and consent to participate

See 'Consent for publication' below.

\section{Consent for publication}

The patient gave his informed consent for publication. This was also documented in his medical records.

\section{Competing interests}

The authors declare that they have no competing interests.

\section{Author details}

${ }^{1}$ Department of Clinical Microbiology, Aalborg University Hospital, Hobrovej 18, 9000 Aalborg, Denmark. ${ }^{2}$ Department of Infectious Diseases, Aalborg University Hospital, Hobrovej 18, 9000 Aalborg, Denmark. ${ }^{3}$ Department of Clinical Medicine, Aalborg University, Aalborg, Denmark.

Received: 3 March 2021 Accepted: 12 April 2021

Published online: 23 April 2021

\section{Reference}

1. Totten PA, Fennell CL, Tenover FC, Wezenberg JM, Perine PL, Stamm WE, Holmes KK. Campylobacter cinaedi (sp. nov.) and Campylobacter fennelliae (sp. nov.): two new Campylobacter species associated with enteric disease in homosexual men. J Infect Dis. 1985:151:131-9.

2. Vandamme P, Falsen E, Rossau R, Hoste B, Segers P, Tytgat R, De Ley J. Revision of Campylobacter, Helicobacter, and Wolinella taxonomy: emendation of generic descriptions and proposal of Arcobacter gen. nov. Int J Syst Bacteriol. 1991:41:88-103.

3. Fernandez KR, Hansen LM, Vandamme P, Beaman BL, Solnick JV. Captive rhesus monkeys (Macaca mulatta) are commonly infected with Helicobacter cinaedi. J Clin Microbiol. 2002:40:1908-12.

4. Gebhart CJ, Fennell CL, Murtaugh MP, Stamm WE. Campylobacter cinaedi is normal intestinal flora in hamsters. J Clin Microbiol. 1989;27:1692-4.

5. Fennell CL, Totten PA, Quinn TC, Patton DL, Holmes KK, Stamm WE. Characterization of Campylobacter-like organisms isolated from homosexual men. J Infect Dis. 1984;149:58-66.

6. Menard A, Smet A. Review: Other helicobacter species. Helicobacter. 2019;24(Suppl 1):e12645.

7. Araoka H, Baba M, Kimura M, Abe M, Inagawa H, Yoneyama A. Clinical characteristics of bacteremia caused by Helicobacter cinaedi and time required for blood cultures to become positive. J Clin Microbiol. 2014;52:1519-22.

8. Kawamura Y, Tomida J, Morita Y, Fujii S, Okamoto T, Akaike T. Clinical and bacteriological characteristics of Helicobacter cinaedi infection. J Infect Chemother. 2014;20:517-26.

9. Araoka H, Baba M, Okada C, Kimura M, Sato T, Yatomi Y, Moriya K, Yoneyama A. First evidence of bacterial translocation from the intestinal tract as a route of Helicobacter cinaedi bacteremia. Helicobacter. 2018;23:e12458.

10. Nielsen HL, Ejlertsen T, Nielsen H. Polycarbonate filtration technique is noninferior to mCCDA for isolation of Campylobacter species from stool samples. Diagn Microbiol Infect Dis. 2015;83:11-2.

11. Rimbara E, Mori S, Matsui M, Suzuki S, Wachino J, Kawamura Y, Shen Z, Fox JG, Shibayama K. Molecular epidemiologic analysis and antimicrobial resistance of Helicobacter cinaedi isolated from seven hospitals in Japan. J Clin Microbiol. 2012;50:2553-60.

12. Kaas RS, Leekitcharoenphon P, Aarestrup FM, Lund O. Solving the problem of comparing whole bacterial genomes across different sequencing platforms. PLoS One. 2014;9:e104984.

13. Holst H, Andresen K, Blom J, Hojlyng N, Kemp M, Krogfelt KA, Christensen $\mathrm{JJ}$. A case of Helicobacter cinaedi bacteraemia in a previously healthy person with cellulitis. Open Microbiol J. 2008;2:29-31.

14. Nielsen $\mathrm{HL}$, Prag J, Krogfelt KA. Helicobacter cinaedi knee infection after arthroscopy in an immunocompetent patient. BMJ Case Rep. 2015. https://doi.org/10.1136/bcr-2014-208637. 
15. Lastovica AJ. Emerging Campylobacter spp.: the tip of the iceberg. Clin Microbiol Newsl. 2006;28:49-56.

16. Araoka H, Baba M, Okada C, Kimura M, Sato T, Yatomi Y, Moriya K, Yoneyama A. Risk factors for recurrent Helicobacter cinaedi bacteremia and the efficacy of selective digestive decontamination with kanamycin to prevent recurrence. Clin Infect Dis. 2018;67:573-8.

17. Sawada O, Gotoh Y, Taniguchi T, Furukawa S, Yoshimura D, Sasaki S, Shida H, Kusunoki Y, Yamamura T, Furuya K, Itoh T, Horita T, Hayashi T, Misawa N. Genome Sequencing Verifies Relapsed Infection of Helicobacter cinaedi. Open Forum Infect Dis. 2019;6:ofz200.

18. Tanaka Y, Koshikawa T, Hamada I, Shibuya K, Watanabe K, Obata A, Takahashi H. Relapsing Helicobacter cinaedi bacteremia in a non-immunocompromised patient. Nihon Naika Gakkai Zasshi. 2011;100:1385-7.

19. Uçkay I, Garbino J, Dietrich PY, Ninet B, Rohner P, Jacomo V. Recurrent bacteremia with Helicobacter cinaedi: case report and review of the literature. BMC Infect Dis. 2006;6:86

20. Ito K, Yamamoto T, Nishio H, Sawaya A, Murakami M, Kitagawa A, Matsuo Y, Matsuo K, Tanaka S, Mori N. Bacteremic kidney cyst infection caused by Helicobacter cinaedi. CEN Case Rep. 2016;5:121-4.

21. Mandai S, Kasagi Y, Kusaka K, Shikuma S, Akita W, Kuwahara M. Helicobacter cinaedi kidney cyst infection and bacteremia in a patient with autosomal dominant polycystic kidney disease. J Infect Chemother. 2014;20:732-4

22. Suzuki T, Kutsuna S, Tsuboi M, Ota M, Hayakawa K, Ohmagari N. Helicobacter cinaedi hepatic cyst infection with bacteremia. Emerg Infect Dis. 2019;25:603-4
23. Kitamura T, Kawamura Y, Ohkusu K, Masaki T, Iwashita H, Sawa T, Fujii S, Okamoto T, Akaike T. Helicobacter cinaedi cellulitis and bacteremia in immunocompetent hosts after orthopedic surgery. J Clin Microbiol. 2007;45:31-8.

24. Rimbara E, Mori S, Kim H, Matsui M, Suzuki S, Takahashi S, Yamamoto S, Mukai M, Shibayama K. Helicobacter cinaedi and Helicobacter fennelliae transmission in a hospital from 2008 to 2012. J Clin Microbiol. 2013;51:2439-42.

25. Kiehlbauch JA, Brenner DJ, Cameron DN, Steigerwalt AG, Makowski JM, Baker CN, Patton CM, Wachsmuth IK. Genotypic and phenotypic characterization of Helicobacter cinaedi and Helicobacter fennelliae strains isolated from humans and animals. J Clin Microbiol. 1995;33:2940-7.

26. Kawamura Y, Tomida J, Miyoshi-Akiyama T, Okamoto T, Narita M, Hashimoto K, Cnockaert M, Vandamme P, Morita Y, Sawa T, Akaike T. Proposal of Helicobacter canicola sp. nov., previously identified as Helicobacter cinaedi, isolated from canines. Syst Appl Microbiol. 2016;39:307-12.

27. Nukui Y, Chino T, Tani C, Sonobe K, Aiso Y, Tohda S, Koike R, Saito R. Molecular epidemiologic and clinical analysis of Helicobacter cinaedi bacteremia in Japan. Helicobacter. 2020;25:e12675.

\section{Publisher's note}

Springer Nature remains neutral with regard to jurisdictional claims in published maps and institutional affiliations.
Ready to submit your research? Choose BMC and benefit from:

- fast, convenient online submission

- thorough peer review by experienced researchers in your field

- rapid publication on acceptance

- support for research data, including large and complex data types

- gold Open Access which fosters wider collaboration and increased citations

- maximum visibility for your research: over 100M website views per year

At BMC, research is always in progress.

Learn more biomedcentral.com/submissions 